\title{
2 Gamma-Ray Production and Absorption Processes
}

\author{
Roland Diehl
}

\subsection{Introduction to Astronomy with Gamma-Rays}

How do $\gamma$-rays compare with other types of radiation? 'Radiation' in common language describes 'energy packages' which travel on straight paths. 'Electromagnetic radiation' is characterized by variations of electric and magnetic fields in space and time. Another type of 'radiation' are 'cosmic rays', very energetic particles discovered early in the 20th century in the upper atmosphere of the Earth and known to pervade interstellar space. These particles are called 'cosmic radiation' because with their high energies they propagate at the speed of light and in certain aspects behave like photons of similar energies ( $\gamma$-rays)'.

In order to understand the nature of $\gamma$-rays in comparison to other electromagnetic radiation, we remind the reader about 'wave-particle' dualism: We may usefully describe electromagnetic radiation either in terms of a propagating particle with energy ${ }^{1} E=\hbar \omega$ or in terms of waves with wavelength ${ }^{2}$ $\lambda=c / \nu$ with characteristic diffraction and interference patterns. The more appropriate description depends on the energy of the radiation, more precisely on the relation between the wavelength of the radiation and the structural dimensions of the matter it interacts with, i.e., typical distances between atoms, lattice constants, fiber thicknesses, grain sizes, field curvature. ${ }^{3}$ Unlike common 'light', $\gamma$-rays do not reflect off surfaces; they have high penetration power.

The spectrum of observed electromagnetic radiation spans more than 20 orders of magnitued on the frequency scale, ${ }^{4}$ ranging from the low-frequency long-wavelength regime of radio waves $\left(\lambda \simeq 10^{2} \mathrm{~cm}\right)$ through the infrared, optical and ultraviolet regimes up to the high frequency short-wavelength regime of $\mathrm{X}$ and $\gamma$-rays $\left(\lambda \leq 10^{-13} \mathrm{~cm}\right)$. In the regime of $\gamma$-rays, the particle

\footnotetext{
${ }^{1} \hbar=1.05 \times 10^{-34} \mathrm{~J} \mathrm{~s}^{-1}=6.58 \times 10^{-22} \mathrm{MeV}$ s is Planck's (reduced) constant, $h / 2 \pi$; $\omega=\nu / 2 \pi$, with $\nu$ the frequency of the radiation $(\mathrm{Hz})$; per photon $\gamma$-rays are thus far more energetic than, e.g., UV radiation with typical energies of $0.5 \mathrm{eV}$. Energy conversion: $1 \mathrm{eV}=1.602 \times 10^{-19} \mathrm{~J}$.

${ }^{2} c=$ speed of light $=2.998 \times 10^{10} \mathrm{~cm} \mathrm{~s}^{-1}$.

3 Typical structural dimensions are dust grain sizes, $10^{-4} \mathrm{~cm}$; atoms, $10^{-8} \mathrm{~cm}$; atomic nuclei, $10^{-13} \mathrm{~cm}$.

${ }^{4}$ Cosmic ray energies range from $\geq \mathrm{MeV}$ up to $10^{20} \mathrm{eV}$.
} 
description of electromagnetic radiation becomes more appropriate than the wave description, which works well for the less energetic radiation at radio or optical wavelengths. (The general field of high-energy astrophysics is addressed in detail by Longair, 1994, in a two-volume textbook.) This helps us understand the penetration power of $\gamma$-rays: the wavelength of the radiation is short compared to the spacing of the atoms in the material, hence the radiation mainly 'sees' the atom's components, the compact nucleus and the electrons at comparatively large distances (which are seen by $\gamma$-rays as almost independent from the nucleus). The atom is mostly 'empty space' for a $\gamma$-ray photon; interactions occur upon relatively low probability encounters with the nucleus or electrons. It is more appropriate to view $\gamma$-rays as energetic particles, their 'size' being their wavelength. In such a corpuscular description, the energy of the photon is a better characteristic quantity than the wavelength or frequency of the electromagnetic field. Energy is measured in voltage units ' $\mathrm{eV}$ ' for electromagnetic particles (photons), $1 \mathrm{MeV}$ being the energy an electron would have if accelerated by an electrostatic potential of a million volts. The $\gamma$-ray domain is the energy regime above X-rays, ${ }^{5}$ above some $100 \mathrm{keV}$. Gamma-rays with energies up to $\mathrm{TeV}=10^{12} \mathrm{eV}$ have been observed, so that the domain of $\gamma$-rays spans about seven orders of magnitude (in frequency / wavelength / energy). ${ }^{6}$

Materials which should serve as 'mirrors' for X- and $\gamma$-rays would need characteristics which make a wave description (i.e., coherent scattering geometries) applicable for the $\gamma$-ray-material interactions. This becomes virtually impossible below a certain wavelength regime corresponding to X-rays of a few $100 \mathrm{keV}$. The specific instrumental techniques of $\gamma$-ray telescopes are described in Chap 3.

What makes $\gamma$-rays so special that we need space observatories? The above description of the interaction between electromagnetic radiation and matter tells us that different effects will characterize the absorbing power of the Earth's atmosphere as we go up in frequency from radio waves to $\gamma$-rays (Fig 2.1). Interaction cross sections are maximized when the wavelength of the radiation becomes similar to the size scale of the scattering centers of matter ('resonances'). Radio waves $(\lambda \geq \mathrm{cm})$ thus easily penetrate the atmosphere; ionized-air atmospheric turbulence has much larger typical scales, and atom dimensions are much smaller. At optical frequencies, the electronic shells of atoms and molecules resonate with electromagnetic radiation to efficiently absorb photon energy - the complex structure of the atmospheric absorption reflects the species' characteristics, molecules and water vapor absorbing

\footnotetext{
${ }^{5}$ Useful criteria could be, e.g., the transition from atomic to nuclear physics at tens of $\mathrm{keV}$, or the rest mass of the electron of $511 \mathrm{keV}$, or the experimental techniques being beyond focussing optics for $\gamma$-rays above a few $100 \mathrm{keV}$. Any such lower limit for $\gamma$-rays corresponds to a wavelength of $5 \times 10^{-5} \mu \mathrm{m}$ or smaller.

${ }^{6}$ For comparison, our eyes see the optical regime of about $0.4-0.7 \mu \mathrm{m}$ wavelength, from colors blue to red - a fairly modest dynamical range.
} 


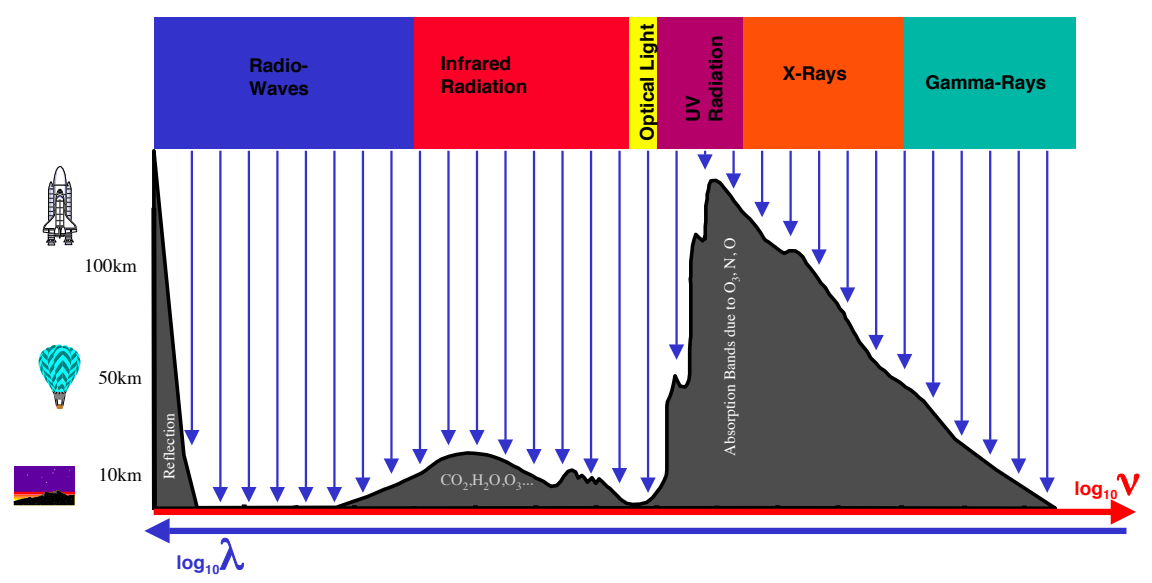

Fig. 2.1. The Earth's atmospheric transparency to cosmic electromagnetic radiation varies considerably over the range of frequencies. Transparency of the atmosphere to radio waves makes radio astronomy a ground-based discipline. Atmospheric dust, water vapor, and molecules block radiation and/or provide a bright atmospheric source in the infrared regime, which means that astronomical observations have to be made from airplanes or spacecraft. There is a narrow window again in the optical regime which allows classical optical astronomy with groundbased telescopes. This window is closed by atomic absorption in the UV through $\mathrm{X}$-rays, making space-based telescopes a necessity. Also beyond, in $\gamma$-rays, continuum scattering and absorption processes with atmospheric electrons and nuclei prevent ground-based astronomy up to very high energies. Only above $\sim \mathrm{TeV}$ energies $\left(>10^{12} \mathrm{eV}\right)$ can electromagnetic showers induced by cosmic $\gamma$-rays in the atmosphere be observed from the ground; indirect information about the primary $\gamma$-ray is retained, and thus ground-based astronomy becomes feasible again

in the infrared regime, atoms like oxygen and nitrogen absorbing strongly in the ultraviolet regime. A 'window' remains in what we call 'visible' light. For more energetic X-ray photons, the inner electrons of atoms have characteristic binding energies similar to the photon energies, and photon interactions with these may absorb X-ray radiation with characteristic spectral absorption edges. Into the $\gamma$-ray regime, scattering off individual electrons is the prime attenuation effect for a $\gamma$-ray beam, a process with intrinsically continuous spectral characteristics, and reduced in strength as the $\gamma$-rays become more energetic. This leads to an 'optical depth' in $\gamma$-rays determined by the scat- 
tering cross section of $\gamma$-rays, described by the Thompson scattering cross section $\sigma_{T}$ in the limit of low energies, ${ }^{7}$

$$
\sigma_{T}=\frac{8 \pi}{3} \cdot r_{e}^{2}
$$

with $r_{e}=2.8 \times 10^{-13} \mathrm{~cm}$, the 'classical electron radius'. At $\mathrm{MeV} \gamma$-ray energies, nuclear interactions become important, and spectral absorption characteristics derive from nuclear structure. Scattering off electrons (and then significantly also off protons) becomes inelastic above the X-ray regime with transfer of energy to the particle ('Compton scattering'), and is more forwardbeamed than Thompson scattering; the decreasing electron scattering cross section in the $\gamma$-ray regime is described by the Klein-Nishina formula: ${ }^{8}$

$$
\sigma_{K N}=r_{e}^{2} \cdot \frac{\pi m_{e} c^{2}}{E_{\gamma}} \cdot\left[\ln \left(\frac{2 E_{\gamma}}{m_{e} c^{2}}+\frac{1}{2}\right)\right] .
$$

The different 'windows' from the surface of the Earth into space determine the techniques used for astronomy: optical, radio, and very-high $\gamma$-ray astronomy can be ground-based, while spaceborne instrumentation is required for ultraviolet, X-ray, $\gamma$-ray and infrared telescopes.

The relative penetration power of $\gamma$-rays derives from the absence of atomic interactions. In a 'thick' layer of material a $\gamma$-ray will however eventually collide with an individual atomic nucleus or electron in spite of their relatively large spacing. The $\gamma$-ray's arrival direction gets lost in repetitive interactions, the energy being redistributed among secondary particles and photons. The penetration depth for $\gamma$-rays corresponds to a few grams of material per $\mathrm{cm}^{2}$. For a characteristic thickness of the Earth's atmosphere of 10 $\mathrm{km}$, and a typical density of air of $1 \mathrm{mg} \mathrm{cm}^{-3}$ this amounts to $1000 \mathrm{~g} \mathrm{~cm}^{-2}$. Thus, the Earth's atmosphere is a thick shield! Gamma-ray telescopes must therefore be operated at high altitudes, above $40 \mathrm{~km}$, to be able to measure cosmic $\gamma$-rays directly. ${ }^{9}$

\subsection{Production Processes}

What are the processes that make $\gamma$-rays? (See also the general textbooks on celestial $\gamma$-rays by Fichtel and Trombka, 1997; Stecker, 1971; Paul and

$\overline{7} E_{\gamma} / m_{e} c^{2} \ll 1$, hence at the upper end of the X-ray regime $\left(m_{e} c^{2}=0.511\right.$ $\mathrm{MeV})$. The Thompson cross section corresponds to the geometrical cross section associated with the classical electron radius $r_{0}=e^{2} / m_{e} c^{2}, \sigma_{T}=8 \pi r_{0}^{2} / 3=$ $0.665 \times 10^{-24} \mathrm{~cm}^{2}$

${ }^{8}$ Here in the high-energy limit $\mathrm{E}_{\gamma} / m_{e} c^{2} \gg 1$.

9 Additionally, the cosmic-ray nuclear interactions with the gas of the Earth's upper atmosphere result in its bright glow with $\gamma$-rays, much brighter than any cosmic sources. Instruments for cosmic $\gamma$-rays have to avoid viewing this luminous layer at $25-30 \mathrm{~km}$ altitude and be adequately constructed to not confuse $\gamma$-rays from the Earth's atmosphere below with $\gamma$-rays from celestial sources. 
Laurent, 1998, for more details.) Electromagnetic radiation may be 'thermal' or 'nonthermal'. Thermal radiation emerges from a large population of electromagnetically interacting particles in equilibrium, with their mean energy characterized by particle temperature. The spectrum of radiation intensity follows the 'blackbody' distribution, which reflects the dynamical equilibrium of state population in the radiation field and the multi-particle system:

$$
I(\nu)=\frac{8 \pi h \nu^{3}}{c^{3}} \cdot \frac{1}{e^{h \nu / k T}-1}
$$

is the energy density ${ }^{10}$ of radiation at frequency $\nu$, which is a function of particle temperature $T$ and Boltzmann's constant $k$. Collisions and coupling interactions between radiating material and radiation are so intense that the energy densities of both are identical. When the 'fire' becomes hotter, the blackbody distribution shifts its median towards more energetic radiation, so that each of the photons carries more energy. From Wien's law, the product of temperature and the wavelength of the peak of the radiation spectrum is a constant: $0.2898(\mathrm{~cm} \mathrm{~K})=\lambda_{\max } \cdot T$. From this relation, $\lambda_{\max }$ falls into the visible regime for $\sim 6000 \mathrm{~K}$, the approximate temperature of the sun's surface, the big bang residual radiation at $2.7 \mathrm{~K}$ temperature peaks at a wavelength below a millimeter. For thermal $\gamma$-rays of $1 \mathrm{MeV}$, the corresponding temperature of a fireball would be above $2 \times 10^{9} \mathrm{~K}^{11}$

Nonthermal processes are thus more typical sources of $\gamma$-rays, with specific interactions of matter fields and radiation. A physical process may generate a $\gamma$-ray, but we now do not require that the entire source environment is thermalized and thus emits blackbody radiation where this $\gamma$-ray emission dominates all radiation processes. In general, all elementary particles which take part in an electromagnetic interaction may be sources of $\gamma$-rays, if accelerated in some way through external fields of any kind. Likewise, if a system of particles (including electromagnetically interacting particles) changes its state to another one with different energy, the energy difference (or parts thereof) may be radiated in the form of electromagnetic field quanta, hence also $\gamma$-rays.

Several processes can be distinguished as shown in Fig. 2.2. ( For a more thorough treatment of electromagnetic-field interactions see textbooks by Jackson, 1999; Rybicki and Lightman, 1979; Rauch and Rohrlich, 1980; Heitler, 1960).

\section{Charged Particles in Strong Electric or Magnetic Fields}

The motion of a charged particle (e.g. electron) can be viewed as a charge current along its trajectory. The particle's charge produces a Coulomb field; its

${ }_{10}$ Per surface area of the black body, $\left(\mathrm{Ws} \mathrm{cm}^{-2}\right)$

${ }^{11}$ Nuclear fusion inside the sun occurs at $15 \times 10^{6} \mathrm{~K}$, corresponding to keV's in thermal energy. In comparison, $\gamma$-ray fireballs would be even hotter. 


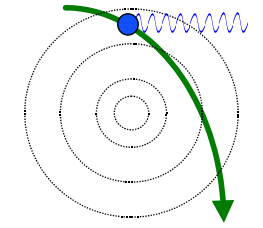

Accelerated Charged Particles

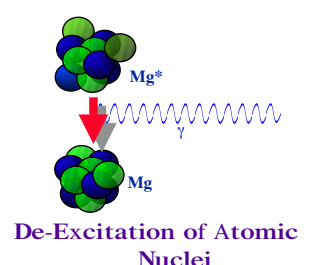

Nuclei
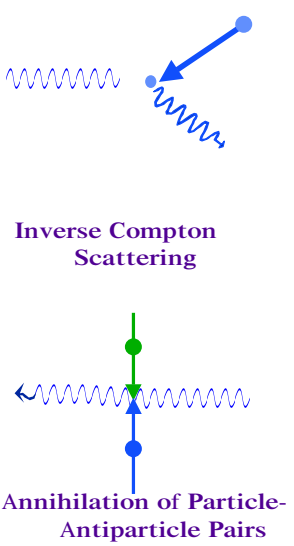

Fig. 2.2. Charged particle acceleration results in photon emission. As an example for charged-particle acceleration the case of bremsstrahlung is illustrated (top left). Weak decays inside nuclei result in excited nuclear states, which often decay through $\gamma$-ray emission (bottom left). Likewise, the decay of unstable particles such as pions, and the annihilation of particle-antiparticle pairs constitute $\gamma$-ray source processes (bottom right). Soft photons of energies lower than $\gamma$-ray energies may gain energy from electromagnetic-field interactions. The inverse-Compton process on energetic electrons or protons is illustrated (top right) as the most important example

motion thus corresponds to an electromagnetic field, varying as the charged particle moves. Any acceleration of the charged particle now can be viewed as dynamic modification of this electromagnetic field, at the expense of the charged particle's energy of motion. Thus kinetic energy is transformed into energy of the electromagnetic field. Again, for the high energies of such electromagnetic energy loss into $\gamma$-rays, the particle picture becomes more appropriate, and quanta of electromagnetic energy, the $\gamma$-ray photons, are emitted by accelerated charged particles, 'particle' momenta being conserved.

The motion of a charged particle in a magnetic field $B$ is described by its 'pitch angle' $\theta$, the angle between the particle trajectory and the direction of the magnetic field, and can be described as gyration of the particle around the field direction, with characteristic gyration frequency $\nu_{g}=e B / 2 \pi m_{e}$. The radiated energy originates from the velocity component perpendicular to the magnetic field, the acceleration of the charged particle being the vec- 
tor product $v \otimes B$. The resulting synchrotron photon spectrum peaks at a frequency

$$
\nu_{c}=\frac{3}{2} \gamma^{2} \nu_{g} \sin \theta=\frac{3}{2} \gamma^{2} \cdot \frac{e B}{2 \pi m_{e} \cdot \sin \theta} .
$$

Energetic electrons $(\simeq 1000 \mathrm{MeV})$ moving in the interstellar magnetic field ${ }^{12}$ thus radiate 'synchrotron' photons, which can be observed in the radio regime. ${ }^{13}$ As a scaling estimate, a high-energy (cosmic ray) electron in a typical interstellar magnetic field would radiate synchrotron photons at

$$
E_{h \nu} \simeq 0.05 \cdot\left(\frac{E_{e}}{(T e V)}\right)^{2} \cdot \frac{B}{(3 \mu G)}(e V)
$$

Both higher magnetic field strength or more energetic particles shift this radiation up in energy, into optical regimes for particle accelerators in highenergy physics laboratories, and into the $\gamma$-ray regime near the surface of neutron stars, ${ }^{14}$ where magnetic fields are many orders of magnitude higher.

Even the curvature of magnetic field lines in the vicinity of neutron stars can provide sufficient 'bending' acceleration to charged particles that move along these field lines, so that 'curvature $\gamma$-rays' are emitted. In pulsars, where magnetic fields are $10^{10} \mathrm{G}$ and more, we attribute part of the observable $\gamma$ rays to this process.

Charged-particle acceleration through electric fields is more important for production of $\gamma$-rays and results in 'bremsstrahlung' emission. For example, an electron passing very close by an atomic nucleus will experience the strong positive charge of the nucleus, so that the electron's trajectory is substantially changed by such acceleration. From this change in electron energy due to the electron-ion collision, we obtain the total intensity per unit frequency in bremsstrahlung radiation ${ }^{15}$

$$
I_{\nu}\left(E_{e}\right)=\frac{Z^{2} e^{6} n}{12 \pi^{3} \epsilon_{0}^{3} c^{3} m_{e}^{2} v_{e}} \cdot \ln \left(\frac{192 v_{e}}{Z^{1 / 3} c}\right) .
$$

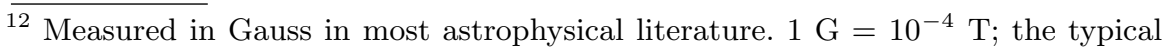
magnetic field strength in the interstellar medium is a few $\mu \mathrm{G}$.

${ }^{13}$ In extreme cases such as young supernova remnants, synchrotron emission may occur in the UV/X-ray regime

${ }^{14}$ In magnetic fields above a critical strength of $\sim 10^{13} \mathrm{G}$, relativistic effects and the quantum phenomena of pair creation and multi-photon states result in a more complex description of the radiation field, called 'magneto-bremsstrahlung' or 'synchro-curvature radiation' (see Chap 6).

${ }^{15} I_{\nu}\left(E_{e}\right)$ is integrated over the collision process, in units of $\mathrm{erg} \mathrm{cm}^{-2}$. We assume the much heavier ion is at rest. $\mathrm{e}=1.602 \times 10^{-19} \mathrm{C}$ is the electron's charge, $v_{e}$ the electron velocity and $m_{e}$ its mass, $Z \cdot e$ the ion charge, $n$ the number density of matter, and $\epsilon_{0}=8.8542 \times 10^{-3} \mathrm{C}^{2} \mathrm{erg}^{-1} \mathrm{~cm}^{-1}$ the dielectric constant or permittivity of the vacuum. 
The spectrum of bremsstrahlung radiation remains flat up to roughly the electron kinetic energy, i.e. up to ${ }^{16}$

$$
E_{\gamma}=(\gamma-1) m_{e} c^{2} .
$$

It drops sharply towards zero above, as effectively all the kinetic energy of the electron has been transferred to the bremsstrahlung photon.

Depending upon the energy distribution of electrons and ions, one distinguishes 'thermal' and 'nonthermal' bremsstrahlung again; this specifies whether the charged-particle energy distribution has been thermalized through collisions, or if 'nonthermal' and reflecting, e.g., a particle-acceleration process such as Fermi acceleration. For a hot plasma, the specific radiation power or emissivity ${ }^{17}$ in thermal bremsstrahlung may be expressed as a function of temperature

$$
k_{\nu}=5.443 \times 10^{-39} \cdot Z^{2} \cdot g(\nu, T) \cdot \exp \left(-E_{\gamma} / k T\right) \cdot T^{-1 / 2} \cdot n_{e} n_{p} .
$$

The 'Gaunt factor' $g(\nu, T)$ encodes the characteristic collision impact parameter distribution for a plasma of specific composition and temperature, and may be approximated in the $\gamma$-ray regime by $\left(E_{\gamma} / k T\right)^{1 / 2}$.

For nonthermal particle spectra, electron energy distributions of a powerlaw type

$$
d N_{e}(E) / d E \sim E^{-\alpha}
$$

are frequently found, with $\alpha \simeq 1-3$. Depending upon energy, ion-electron bremsstrahlung dominates electron-electron bremsstrahlung at low energies, while in the high-energy $\gamma$-ray regime the ratio of both rises to order unity. Inverse bremsstrahlung, which is the photon emission from energetic protons and ions colliding with ambient electrons or ions, is negligible in most circumstances.

Summarizing, the acceleration of charged particles of high energy by a suitable force field may result in $\gamma$-rays. Hence, observation of $\gamma$-rays may be used to study energetic particles moving in strong fields. 'Energetic' here means travelling almost at the speed of light with $\gg \mathrm{MeV}$ energies; fields must be strong enough to change the particle's motion by a significant amount.

\section{Inverse Compton Scattering}

Up-scattering of photons of lower energy through collisions with energetic particles is termed the 'Inverse-Compton process'. As we employ the 'particle' description for $\gamma$-rays, we may imagine them to collide with other particles

16 With the electron's Lorentz factor $\gamma=\frac{1}{\sqrt{1-\frac{v^{2}}{c^{2}}}}$.

17 Per unit frequency, in $\mathrm{W} \mathrm{cm} \mathrm{cm}^{-3} \mathrm{~Hz}^{-1}$, using $T^{\mathrm{c}^{2}}$ for the temperature of the plasma in $\mathrm{K} ; \mathrm{k}=8.6174 \times 10^{-5} \mathrm{eV} \mathrm{K}^{-1}$ is Boltzmann's constant, and $n$ the electron and ion number density per $\mathrm{cm}^{3}$. 
such as electrons. This is known as the Compton scattering process. Equivalently, we may also view the electromagnetic field which corresponds to a photon to penetrate into the regime of the strong electromagnetic field caused by the charged particle ('wave' or 'field' description). In 'normal' Compton scattering a $\gamma$-ray photon will collide with one of the many atomic electrons within some material and be scattered in the collision, transferring some of its energy to the electron. The inverse energetics may also apply, however, and provide a process for $\gamma$-ray production: if photons of lower energy collide with energetic electrons, these photons may gain energy in the collisions, thus being promoted in energy, e.g., from X-rays to $\gamma$-rays. This 'inverse-Compton scattering' is important in regions of high photon densities. Other examples are compact stars where an accretion disk is sufficiently hot to emit X-rays, and the compact object generates beams of charged particles in its vicinity (see below). The spectral intensity distribution of inverse-Comptonized isotropic photons of frequency $\nu_{0}$ and photon density $N\left(\nu_{0}\right)$ from scattering off high-energy electrons of Lorentz factor $\gamma$ is

$$
j_{\nu}\left(\nu_{0}\right)=\frac{3 \sigma_{T} c}{16 \gamma^{4}} \cdot \frac{N\left(\nu_{0}\right)}{\nu_{0}^{2}} \cdot \nu \cdot\left[2 \nu \cdot \ln \left(\frac{\nu}{4 \gamma^{2} \nu_{0}}\right)+\nu+4 \gamma^{2} \nu_{0}-\frac{\nu^{2}}{2 \gamma^{2} \nu_{0}}\right]
$$

with $\sigma_{T}$ as the Thompson scattering cross section. ${ }^{18}$ The typical energy of inverse-Compton-scattered photons rises rapidly with energy:

$$
E_{\gamma} \simeq 1.3\left(\frac{E_{e}}{(T e V)}\right)^{2} \cdot\left(\frac{E_{p h}}{2 \times 10^{-4}(e V)}\right)(G e V),
$$

with an ambient photon-field ${ }^{19}$ typical energy $E_{p h}$. Therefore the inverseCompton process plays an important role for cosmic $\gamma$-ray sources. The blackbody thermal radiation of the ambient field may be expressed as the energy distribution of photons, as number density (photons $\mathrm{cm}^{-3}$ ) of ambient photons with energy $E=h \nu$,

$$
n_{B B}\left(E_{h \nu}\right)=\frac{\left(E_{h \nu} / m_{e} c^{2}\right)^{2}}{\pi^{2} \lambda_{c}^{3}} \cdot \frac{1}{\exp \left(E_{h \nu} / k T\right)-1},
$$

using the definition of the Compton wavelength $\lambda_{C}=h / 2 \pi m_{e} c$. These photons may be up-scattered to $\gamma$-ray energies by collision with high-energy electrons of energy $E_{e}$.

\section{Nuclear Transitions}

The nuclear (or strong) force is responsible for the binding of protons and neutrons in the atomic nucleus. It outweighs the Coulomb repulsion between

\footnotetext{
18 At high energies, energy transfer to the electron may become important. This will imply treatment through the Compton scattering formalism, replacing the Thompson cross section with the Klein-Nishina expression.

19 Photons from the cosmic $3 \mathrm{~K}$ background radiation are characterized by a typical energy $2 \times 10^{-4} \mathrm{eV}$ and energy density of $0.26 \mathrm{eV} \mathrm{cm}^{-3}$.
} 
the protons closely packed in the nucleus. As a quantum system, the nucleus has specific, quantized states of energy for this compact assembly of nucleons, which can be thought of to be similar than states of electrons in atomic shells, by analogy. ${ }^{20}$ These nuclear states have typical energy spacings of $\sim \mathrm{MeV}$; hence any transition in states of atomic nuclei may involve absorption or emission of $\mathrm{MeV} \gamma$-rays,

$$
{ }^{n} X \longrightarrow{ }^{n-1} Y^{*}+e^{+} \longrightarrow{ }^{n-1} Y+\gamma \text {. }
$$

Therefore whenever some energetic interaction brings disorder into the state of a nucleus, we expect $\gamma$-ray line emission from nuclear de-excitation. Energetic collisions from cosmic rays with interstellar gas nuclei are one example, radioactive decay of a freshly synthesized nucleus another. In radioactive decay, one of the particles of the nucleus transforms into one of another kind as a consequence of the 'weak force'; neutrons decay into protons (' $\beta^{-}$-decay'), or protons can transform into neutrons (' $\beta^{+}$-decay'). The so-caused disorder in the tight arrangement of nucleons is unstable under the influence of the strong and electric forces, and new, stable arrangements are obtained, through emission of the energy difference, often as a $\gamma$-ray in the $\mathrm{MeV}$ regime. Some characteristic energy levels of first-excited states which are important for $\gamma$-ray astronomy are $4.438 \mathrm{MeV}\left({ }^{12} \mathrm{C}^{*}\right), 6.129 \mathrm{MeV}\left({ }^{16} \mathrm{O}^{*}\right)$, and 1.809 $\mathrm{MeV}\left({ }^{26} \mathrm{Mg}^{*}\right)$. The cross section for excitation of nuclei into these levels is maximized in resonances, i.e. when the collision energy is in the vicinity of the energy of the excited level. This implies that low-energy cosmic-ray interactions become visible in the domain of $\gamma$-ray lines. Generally, observation of characteristic $\gamma$-ray lines thus tell us about nuclear transitions in a region of either nucleosynthesis or cosmic-ray interactions.

\section{Decays and Annihilation}

Pion decay is somewhat similar to above nuclear processes. The pion is an elementary particle involved in the strong nuclear interaction. Pions are created during strong-interaction events such as collisions of cosmic-ray protons with ambient-gas nuclei. Neutral pions $\left(\pi^{o}\right)$ decay rapidly into two $\gamma$-ray photons, with an energy distribution peaking at $\sim 70 \mathrm{MeV}$, half the rest mass of the pion. Observation of a pion decay peak in a $\gamma$-ray spectrum thus provides insight into collisions of energetic $(>135 \mathrm{MeV})$ protons with nuclei. The pion decay $\gamma$-ray bump is broadened as the momentum distribution of

20 This corresponds to the shell model of the nucleus; for heavier nuclei a drop model better describes the nucleus (see nuclear physics literature for details). In atomic shell transitions between electron states, familiar line radiation is emitted. Inelastic collisions of unbound electrons in the vicinity of nuclei may also result in photon emission; this process is called 'free-free emission', from scattering of the electrons between 'free' or unbound atomic states; as opposed to 'bound-free' transitions, which emit UV/X-ray line radiation. 
the high-energy collision adds a Doppler shift and broadening. For beamed annihilation (e.g. in jets), the peak will be Doppler-shifted towards higher energies.

Annihilation of pairs of particle and anti-particle also produces $\gamma$-rays. From equivalence of mass and energy, field energy may be converted into pairs of particles and anti-particles in sufficiently strong electromagnetic fields. Even vacuum field fluctuations would produce virtual pairs of particles and antiparticles; however in a strong field, the trajectories of both particles diverge sufficiently such that the inverse 'annihilation process' does not occur immediately. The lightest particle-antiparticle pair that can be generated is the electron and its antiparticle, the positron, each with $511 \mathrm{keV}$ rest mass; creation requires a minimum energy of $1.022 \mathrm{MeV}$. The inverse process occurs when a particle encounters its antiparticle, and is called 'annihilation'; the mass of both particles is radiated as electromagnetic energy. Conservation laws demand that electron-positron annihilation will produce two (or more) photons. Distribution of the rest mass energy among these photons results in $0.511 \mathrm{MeV}$ photons for two-photon annihilation, in the rest frame of the annihilation process. Positrons and electrons also may form a bound, atomlike system, which exists in two different states distinguished by the relative orientations of particle spins. The parallel-spin state forms a triplet in external fields and decays into three photons because of spin and momentum conservation, so that three photons share the $1.022 \mathrm{MeV}$ total annihilation energy; this produces a $\gamma$-ray spectrum with a maximum energy of $511 \mathrm{keV}$ and a continuum distribution towards lower energies.

Radioactive decay processes involving ' $\beta$-decays' also produce posi-trons. Through the weak interaction, a proton can decay into a positron and a neutron (plus an electron-neutrino $\nu_{e}$, but we ignore this highly penetrating particle here).

Annihilation photons originate in the vicinity of radioactive decay regions, or energetic environments capable of positron production by other processes. The surface and vicinity of compact stars (neutron stars and black holes) may have such high energy density both from gravity and from strong magnetic fields compressed from the original star. Similarly, hadronic antiparticles (antiprotons and antimatter nuclei) in the universe may annihilate upon collision with normal matter and be responsible for spectral features at correspondingly higher energies in the $\gamma$-ray spectrum.

\section{Charged particles bound in strong magnetic fields}

Just as the electric (or Coulomb) force holds electrons close to a nucleus in the quantized system of an atom, strong magnetic fields can force electrons into orbits around their field lines, leading to quantized energy levels for allowed electron energies. Magnetic fields close to neutron stars can be sufficiently strong for the steps between such allowed electron orbit levels to be in the regime of tens of $\mathrm{keV}$, the low $\gamma$-ray regime (or high $\mathrm{X}$-ray regime). 
Electron transitions from one allowed state to another will eject or absorb photons of this characteristic energy difference, producing so-called cyclotron line radiation. Note that only the motion perpendicular to the magnetic field is quantized, the parallel velocity component is unaffected by the magnetic field. The 'Landau' energy levels $E=h \nu_{c}$ may be obtained from the cyclotron frequency

$$
\nu_{c}=Z e B / 2 \pi \gamma m_{0},
$$

for a particle with charge $Z e$ and velocity $v$ [hence with a Lorentz factor $\left.\gamma=1 / \sqrt{1-\left(v^{2} / c^{2}\right)}\right]$ in a magnetic field $B$. For field strength values around $10^{12} \mathrm{G}$ as observed in strongly magnetized neutron stars, cyclotron lines fall in the X-ray regime, ${ }^{21}$ from $\hbar \omega \simeq 12 \mathrm{keV} B /\left(10^{12} \mathrm{G}\right)$.

We have seen that the processes which create $\gamma$-rays are different in general from the more familiar thermal blackbody radiation. The relevant physical processes involve rather special conditions. Generally speaking, violent processes are at play. Observation of $\gamma$-rays enables us to study such exceptional places in nature and thus explores a different aspect of the universe than optical observation.

\subsection{Gamma-Ray Interactions, Absorption Processes}

How does a $\gamma$-ray interact with matter? From the above description, we learn that, unlike optical light which is deflected from mirror surfaces, $\gamma$-rays do not interact with the surface of materials. The $\gamma$-rays mainly scatter off electrons within materials, and they randomly penetrate to some variable depth of material before their interaction. Scattering of $\gamma$-rays is itself a random process with a wide spectrum of possible results, averaging to the distributions presented above when many interactions are summed; the individual fate of a $\gamma$-ray photon in a material is not predictable. Moreover, scattering of incident $\gamma$-ray photons will generally be incoherent, i.e. the scattered photons are unrelated, and do not form, e.g., patterns of diffraction. The wave description of electromagnetic interactions with matter is inappropriate; we apply a particle interaction model instead.

The interaction processes vary with the energy of the $\gamma$-ray, and are as follows (from low to high energy; see Fig 2.2):

- Photoelectric absorption: Atomic electrons are removed from their nuclei, thus reducing the energy of the $\gamma$-ray photon by the electron's binding energy. This is the dominant process at energies below $100 \mathrm{keV}$.

- Compton scattering: Electrons are hit by the $\gamma$-ray photons, and gain a fraction of the photon's kinetic energy in this collision. This is the dominant interaction process in the $0.1 \mathrm{MeV}$ to a few $\mathrm{MeV}$ regime.

$\overline{21}$ The cyclotron frequency of a non-relativistic electron $(\gamma=1)$ is $2.8 \mathrm{MHz} \mathrm{G}^{-1}$. 
- Pair creation: In the presence of an electric field (usually of the atomic nucleus), the $\gamma$-ray energy may be converted into a particle-antiparticle pair, electron and positron. From momentum conservation, these two particles move in opposite directions so that the momentum component perpendicular to the $\gamma$-ray cancels; transformation into the laboratory system makes them appear to fork out of the $\gamma$-ray's incidence direction with a narrow opening angle, which decreases with energy. This interaction process cannot occur below a threshold of $1.022 \mathrm{MeV}$, and the cross section increases with energy so that it dominates over Compton scattering above several $\mathrm{MeV}$. The same process can occur in dense photon fields through collisions of energetic photons (Nikischow effect; photon-photon pair production, see also Chap 2.3).

- Quantized absorption: Systems such as atomic nuclei or charged particles captured in strong magnetic fields are characterized by states with level differences in the regime of $\gamma$-ray energies. Hence, state transitions of such systems may absorb $\gamma$-rays resonantly, e.g. forming an excited nucleus. When the reverse transition to the system ground state is inhibited (e.g. from transition selection rules, or high collision frequencies), other interactions may dissipate the excitation energy, e.g., into lattice phonons, and no fluorescent $\gamma$-ray emission occurs; the $\gamma$-ray is absorbed. This process is analogous to formation of optical absorption lines such as Fraunhofer lines.

\subsection{Cosmic Gamma-Ray Sources}

According to the characteristic production processes, we may distinguish the following different types of cosmic $\gamma$-ray sources, as described in subsequent chapters in detail:

- Fireballs, such as found in $\gamma$-ray bursts, and possibly in the vicinity of black holes

- Explosive events of extreme energy density, such as supernovae and novae

- Energetic collisions, such as in the vicinity of accreting compact objects, particle jet sources (microquasars, active galactic nuclei), or cosmic ray collisions with ambient matter, or in solar flares

- Charged-particle beams in the vicinity of compact objects - hence strong gravitational or magnetic fields (quasars, active galactic nuclei)

The thermal radiation from an optically thick ' $\gamma$-ray fireball' constitutes probably the most extreme and violent source from the above. The Big Bang, but also explosions of stars in supernovae (or $\gamma$-ray bursts) in principle approximate this extreme. In explosive events, gigantic amounts of energy may be released within short times (fractions of seconds) into dense environments, and thus provide extreme heat. However, we rarely can see the $\gamma$-ray photosphere of such a fireball due to the scattering and absorption of overlying 
matter surrounding the fireball site. Fireball 'temperatures' may exceed several $10^{9} \mathrm{~K}$ and cause atomic nuclei to dissolve and rearrange upon cooling down, with radioactive nuclei as by-products, whose decay may produce $\gamma$ rays. Direct $\gamma$-ray observations of sufficiently hot fireballs may be possible for events where the energy release is not covered by stellar envelopes, such as neutron star collisions and similarly extreme and rare events, which have been discussed as possible explanations of $\gamma$-ray bursts. Moreover, $\gamma$-rays may provide unique insights to such processes, even though originating from secondary, nonthermal processes, as in the case of radioactive decay.

Neutron stars are highly compact objects, with $\simeq 1$ solar mass compressed into a $10 \mathrm{~km}$-sized sphere. They are known to be common sources of X-rays, mostly caused by release of gravitational energy when matter falls onto their surface. The complex path of matter accreting onto the star in strong gravitational and magnetic fields is the subject of broad astrophysical studies, involving radiation from radio frequencies to $\gamma$-rays. The extreme plasma motions near neutron stars cause complicated accretion disk configurations and beams of particles, and these in turn produce the fascinating diversity of pulsing phenomena of these objects in the X-ray regime. Nuclear excitation of infalling matter from close to the neutron star's surface can be expected to result in characteristic $\gamma$-ray line emission. Further out in the magnetosphere, $\gamma$-rays within a broad frequency range are known to be produced in isolated neutron stars whose magnetosphere is relatively undisturbed by accreting matter. Their $\gamma$-ray emission is attributed to curvature radiation of particles accelerated by large electric fields. The observed pulsing behavior varies strongly with frequency of the radiation and can be explored to diagnose the plasma acceleration and magnetic field configurations in great detail.

Charged-particle accelerators and relativistic-plasma interactions on gigantic dimensions are observed in the extremely luminous nuclei of a subtype of galaxies, the 'active galactic nuclei'. Here particle energies apparently achieve the highest values allowed from the principles of physics, $\gamma$-rays up to $10 \mathrm{TeV}$ have been seen from such objects. Spectacular jets of plasma are ejected from these active galaxies, extending many thousand light years into space. Intense $\gamma$-ray emission has been observed from such galaxies (called therefore $\gamma$-ray blazars) when we view such jets directly (under viewing angles of the jet axis of a few degrees at most). We still do not know what powers the inner cores of these galaxies to make them far more luminous than the entire population of the $10^{10}$ stars of normal galaxies. From detailed studies of nearby accreting compact stars we may learn how gravitational energy can be converted into such jet-like plasma beams. Extreme magnetospheres can be studied in $\gamma$-ray pulsars. Combining these lessons, we may have a better understanding of the extreme phenomena in active galactic nuclei and near black holes.

We speculate that the combined $\gamma$ radiation from active galactic nuclei may comprise the bulk of the diffuse sky brightness in $\gamma$-rays, called the 
cosmic diffuse $\gamma$-ray background. Gamma-ray lines from the radioactivity of cosmological supernovae superimpose (with their characteristic redshift) to add to the cosmic diffuse $\gamma$-ray background in the $\simeq 1 \mathrm{MeV}$ regime; potentially this contribution can be identified from its distinct spectral edges.

The bright large-scale $\gamma$-ray emission observed from our Galaxy is caused by the interaction of cosmic rays with interstellar gas. bremsstrahlung and pion decay processes cause a diffuse glow of the Galaxy in $\gamma$-rays from $\mathrm{MeV}$ energies up to $10^{4} \mathrm{MeV}$, supplemented by inverse-Compton $\gamma$-rays from starlight boosted by cosmic rays. This $\gamma$-ray glow provides a unique tracer to study cosmic rays indirectly throughout the Galaxy. The sources of cosmic rays, in particular the origin of charged particles with energies up to $10^{20}$ $\mathrm{eV},{ }^{22}$, are still unknown. Acceleration in the expanding remnants of supernova explosions has been proposed as the most likely source. The composition of accelerated material is investigated through direct cosmic-ray isotope measurements and nucleosynthesis arguments, to eventually reveal the injection process into such particle acceleration regions. ${ }^{23}$ The Sun itself and the $\gamma$-ray emission from solar-flare particle interactions in the upper solar atmosphere provides a nearby laboratory to study details of such interactions with superior obervational resolution.

\subsection{The Fate of Gamma-Rays Between Source and Detector}

On their journey from the source region to our detectors, $\gamma$-rays may traverse environments with substantial interaction probabilities, both in the vicinity of the source region as well as close to our telescope platform. Absorption and scattering will be parts of our measurement, and we must account for them in our studies.

Low-energy $\gamma$-rays traverse long paths of interstellar space without scattering or absorption. The interstellar gas and dust absorbs optical radiation readily at equivalent column densities of $10^{23}$ hydrogen atoms $\mathrm{cm}^{-2}$ and prevents useful measurements; this much material amounts to less than 0.1 $\mathrm{g} \mathrm{cm}^{-2}$, which is practically transparent to $\gamma$-rays. Such an effective material thickness corresponds roughly to occultation by a sheet of paper: optically, we cannot see through easily, but $\gamma$-rays hardly notice this material. We note however that even in 'empty' intergalactic space, $\gamma$-ray source photons may suffer energy losses over the large distances ${ }^{24}$ from cosmic sources to

22 Ultra-high-energy cosmic-rays (UHECR).

${ }^{23}$ This explanation appears inadequate for energies above $10^{15} \mathrm{eV}$; thus plasmajet-related acceleration sites similar to active galactic nuclei may be needed for higher energies.

24 The nearest galaxies are at distances of $100 \mathrm{kpc}$, while quasar distances are more appropriately expressed in terms of Doppler redshift of spectral lines, expressed as fractional redshift from $z=0$ (= no shift, nearby, $\sim \mathrm{Mpc}$ ) out to $z \simeq 4$, the 


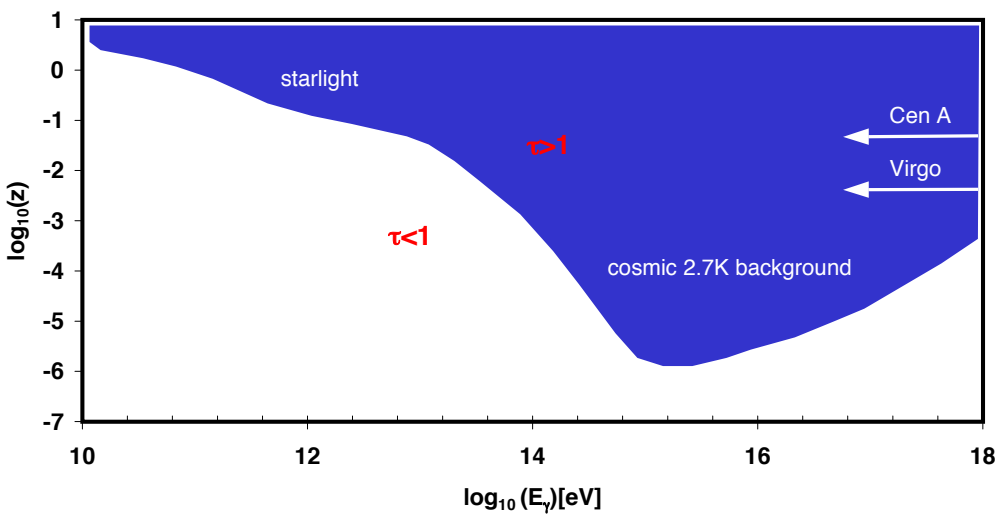

Fig. 2.3. The horizon of high-energy $\gamma$-rays. The shaded region marks the regime of large optical depth, i.e., $\gamma$-rays at these energies from sources at these redshifts will not reach us. The basis for this curve is an ambient photon distribution and intensity composed of the cosmic microwave background and average starlight. The contribution of each component to the absorption from $\gamma-\gamma$-pair production is indicated

$\gamma$-ray telescopes. Over such large distances, even a normally unimportant interaction process may become crucial. On a large scale, the universe is dominated by radiation, with major components from starlight and from the cosmic-diffuse $2.7 \mathrm{~K}$ microwave glow remaining from the Big Bang. Gammaray photons may interact with these ambient photons, producing $\mathrm{e}^{+} \mathrm{e}^{-}$pairs. Therefore the horizon for $\gamma$-rays is limited by this pair production process, despite their penetrating nature. Photon-photon pair production is possible only above a threshold energy given by the rest mass of the pair,

$$
E_{t h-p p}=\frac{2 m_{e}^{2} c^{4}}{[1-\cos (\phi)](1+z)^{2} E_{\gamma}} \sim 1\left(\frac{1+z}{4}\right)^{-2} \cdot \frac{30 G e V}{E_{\gamma}}(e V)
$$

(for head-on collisions, $\phi$ is the photon collision angle). We see that above $\sim 30 \mathrm{GeV}$ the energy loss of $\gamma$-rays in interstellar space from scattering with starlight becomes significant and limits the horizon to $\sim 500 \mathrm{Mpc}$ at $1 \mathrm{TeV}$, while at higher energies scattering on cosmic background photons effectively

most distant quasars observed so far, corresponding to $10^{5} \mathrm{Mpc}$. The details of the conversion from redshift to distance depend on the cosmological model. 
cuts the visibility distance to the few nearest galaxies only (Fig ??). Conversely, sources at distances above $\mathrm{z} \sim 2$ cannot be seen directly above this energy. The $\gamma$-ray horizon is shown in Fig ?? for the upper regime of the $\gamma$-ray domain in terms of redshift of the source. Note that photon-photon pair production results in high-energy charged particles; these will inverse-Compton scatter on the same photons, and redistribute the high-energy $\gamma$-ray energy to lower $\gamma$-ray energies, according to

$$
E_{\gamma, I C} \sim 10 \cdot\left(\frac{1+z}{4}\right)\left(\frac{E_{e}}{30 G e V}\right)(M e V),
$$

thus producing a diffuse-cosmic continuum spectrum in the form of a powerlaw $I \propto E^{-\alpha}$ with index $\alpha \sim 2$.

In dense environments, interactions with charged particles are more important than photon-photon processes, and $\gamma$-rays will mostly scatter off electrons, losing part of their energy to the plasma in this way. This Compton scattering will therefore modify the shape of the spectrum of $\gamma$-rays, and we must account for the plasma conditions in order to reveal the original source $\gamma$-ray spectrum through proper calculation. Note that photons may also gain energy in such plasma collisions, if the plasma is more energetic than the photons; this results in observation of 'Comptonized' $\gamma$ radiation (see Chap 2.2 and Fig 2.2 above). Line features in spectra can be significantly distorted by these effects, and scattering too. We can use such distortions to diagnose the plasma conditions, from the measured profile of $\gamma$-ray lines. Even if unaffected by additional scattering, the line profile will tell us about the relative motion of the $\gamma$-ray source and our observing telescope: Doppler shift of the original frequency tells us about the kinetic energies within the source, such as the expanding motion of radioactive matter after a supernova explosion, translating velocities of $\simeq 1000$ s of $\mathrm{km} \mathrm{s}^{-1}$ into a line width of $\sim 0.1 \mathrm{MeV}$ for a $1 \mathrm{MeV}$ line energy (normally, this line would be narrower than the best instrumental resolution of $\simeq 1 \mathrm{keV}$ ). Note that the gravitational field of compact sources such as neutron star surfaces can also result in substantial changes in line energies: photons have a hard time leaving the star against this strong gravitational force, and can lose $\simeq 20 \%$ of their energy, with a corresponding downward frequency shift.

Getting close to the detector, $\gamma$-rays again encounter material in the upper atmosphere of the Earth. Interactions in the upper atmosphere result in electromagnetic showers with large numbers of secondaries. At very high $\gamma$-ray energies, these are directly used for ground-level detection of the incident $\gamma$-ray. Telescopes for cosmic $\gamma$-ray measurements at lower energies have to operate at least at altitudes above $\sim 40 \mathrm{~km}$, i.e. with residual-atmosphere thicknesses below $\sim 3 \mathrm{~g} \mathrm{~cm}^{-2}$. For these measurements, secondaries produced in the upper atmosphere and within the generally massive spacecraft platform of the telescope provide undesired background. This, and even more so the enormous bombardment of the spacecraft by charged particles from the radiation belts and from cosmic radiation, results in a glow of $\gamma$-rays from 
such spacecraft and instruments. The next chapter illustrates that $\gamma$-ray telescopes have to be built with complex detector configurations and triggering techniques; additionally, powerful analysis algorithms must be employed to discriminate the primary cosmic $\gamma$-rays from the instrumental background. 


\section{Bibliography}

Fichtel C.E. and Trombka J.I.: Gamma-Ray Astrophysics, NASA-1386, 1997

Heitler W.: The Quantum Theory of Radiation, Oxford: Clarendon Press, 1960

Jackson J.D.: Classical Electrodynamics, New York:John Wiley, 1999

Longair M.S.: High-Energy Astronomy, Cambridge:Cambridge University Press, 1994

Paul J. and Laurent P.: Astronomie Gamma Spatiale, Paris:Gordon \& Breach, 1998

Ramana Murthy P.V. and Wolfendale A.W.: Gamma-Ray Astronomy, Cambridge:Cambridge University Press, 1981

Rauch M.M. and Rohrlich F.: The Theory of Photons and Electrons, Berlin:Springer, 1980

Rybicki G.B. and Lightman A.P.: Radiative Processes in Astrophysics, New York:Wiley, 1979

Stecker F.W.: Cosmic Gamma-Rays, NASA SP-249, 1971 